\title{
Salmonella Typhi meningitis in a 9-year old boy with urinary schistosomiasis: a case report
}

\author{
Flora Chacha ${ }^{1 \dagger}$, Stephen E Mshana ${ }^{2 *+}$, Mariam M Mirambo ${ }^{2}$, Martha F Mushi ${ }^{2}$, Rogatus Kabymera' ${ }^{1}$ Lisa Gerwing ${ }^{2}$, \\ Wilhelm Schneiderhan ${ }^{3}$, Ortrud Zimmermann ${ }^{3}$ and Uwe Groß ${ }^{3}$
}

\begin{abstract}
Background: Cases of Salmonella Typhi meningitis have been rarely reported in infants. There are few documented cases of persistent salmonella bacteraemia in patients with schistosomiasis. A presented case highlights the importance of broadening the list of pathogens that can cause meningitis among older children in schistosomiasis endemic regions.

Case presentation: The reported case is of a 9-year old sukuma-black African boy referred to Bugando Medical Centre with complaints of fever, abdominal pain, headache and generalized body weakness. On examination; the child was febrile $\left(39^{\circ} \mathrm{C}\right)$ with neck stiffness and distended abdomen. Cerebrospinal fluid culture was positive for Salmonella Typhi. In addition on urine sediments microscopy, Schistosoma haematobium eggs were seen. The child improved clinically on ceftriaxone and praziquantel, and was discharged 3 weeks after admission.
\end{abstract}

Conclusion: Complicated persistent salmonella infection should be considered in schistosomiasis endemic areas. More research should be done to confirm the association between salmonella infections and urinary schistosomiasis.

Keywords: Meningitis, Salmonella Typhi, Schistosoma haematobium

\section{Background}

Salmonella infections are common causes of febrile illness in sub-Saharan Africa especially in areas where Human Immunodeficiency Virus (HIV) is endemic [1,2]. In addition about $29.1 \%$ of non-malaria bloodstream infections in Africa are due to Salmonella enterica with $58 \cdot 4 \%$ of these due to non-typhoidal Salmonella [3]. In Malawi, non-typhoidal salmonella has been found to be the commonest cause of meningitis in neonates [4]. In infants and young adults, the usual infections due to salmonella include typhoid fever and gastroenteritis. However, in Africa bloodstream infections from nontyphoidal salmonella are common $[4,5]$. Worldwide, few cases of typhoid and non-typhoid salmonella meningitis have been reported in infants [4,6-8]. The reported cases have been associated with increased morbidity and mortality. Mortality rates as high as $40 \%$ have been reported

\footnotetext{
* Correspondence: mshana72@yahoo.com

${ }^{\dagger}$ Equal contributors

${ }^{2}$ Department of Microbiology and Immunology Weill Bugando School of Medicine, CUHAS-Bugando, P.O. BOX 1464, Mwanza, Tanzania

Full list of author information is available at the end of the article
}

in children with salmonella meningitis irrespective of the serotype [9-11]. We present a case of Salmonella Typhi meningitis in a 9-year old boy associated with peritonitis, neutropenia and urinary schistosomiasis.

\section{Case presentation}

A 9-year old sukuma-black African boy was referred to the Bugando Medical Centre (BMC) from a district hospital with three weeks history of fever and abdominal distention and one week history of generalized body weakness and headache. This was the second admission in his life time; the first admission was 4 years ago due to severe malaria. He is $8^{\text {th }}$ child in the family of 10 children and he was fully immunized as per Tanzanian Expanded programme of immunization. There was no history of convulsion, loss of consciousness, vomiting and diarrhea. It was reported that the abdomen was grossly distended and tender. However, he was passing stool normally. Also, it was reported that he used amoxicillin and gentamicin at the district hospital with no improvement. On examination, he was febrile $\left(39^{\circ} \mathrm{C}\right)$, ill looking, pale, and jaundiced. Abdomen was 
grossly distended, tender with shifting dullness. Liver and spleen were not palpably enlarged and there was no rebound tenderness. He had neck stiffness with negative Kernig's signs. Lungs were clear on auscultation, first (S1) and second (S2) heart sounds were heard with a gallop rhythm. Respiratory rate was $46 /$ minute while the heart rate was 120 beats/minute with oxygen saturation of $94 \%$ on ambient air. Provisional diagnosis of severe malaria was reached with differentials of meningitis, peritonitis and typhoid fever.

Lumbar puncture (LP) was done which revealed a xanthochromic cerebrospinal fluid(CSF) with total white blood cells (WBC) count of $52 / \mathrm{cmm} 3$ of which $88 \%$ were lymphocytes. A reactive mononuclear cell was $10 \%$ while pandy's test was positive and cryptococcal antigen test was negative. On CSF gram stain, no organisms were seen. A nasogastric tube was inserted and the child was made nil by mouth due to distended abdomen. Intravenous ceftriaxone $1 \mathrm{~g} 24$ hrly for 2 weeks and intramuscular artemether $64 \mathrm{mg}$ stat then $32 \mathrm{mg}$ daily for 7 days were initiated.

Abdominal ultrasound revealed increased free fluid in the peritoneal cavity, slightly enlarged liver and distended gall bladder with thickened wall (Figure 1). In other laboratory investigations; hemoglobin level of $8.4 \mathrm{~g} / \mathrm{dl}$, total WBC count of $3,100 / \mathrm{mm} 3$ (neutrophils $75 \%$, lymphocyte $19 \%$ ) and platelet count of $181,000 / \mu \mathrm{l}$ were detected.
Widal test results against $\mathrm{O}$ (Salmonella Typhi O) and $\mathrm{H}$ (Salmonella Typhi H) were both $>1: 160$. The rapid Hepatitis B surface antigen, HIV rapid test and blood slide for malaria parasites were all negative. Slightly elevated liver enzymes were noted; alanine transaminase (51.63 U/L) and aspartate transaminase (54 U/L). Bilirubin levels were found to be high with total bilirubin of $176.3 \mu \mathrm{mol} / \mathrm{L}$ and direct bilirubin of $133.5 \mu \mathrm{mol} / \mathrm{L}$.

Urine microscopy was positive for Schistosoma haematobium ova and red blood cells (RBC) (10-20/high power field). On stool microscopy no ova/cysts were seen but numerous white blood cells were seen. CSF culture on blood agar plate (Oxoid Limited, UK) was positive for a significant growth of pure culture of gram negative short rods bacteria. During subsequent culture, growth was also seen on MacKonkey agar (Oxoid Limited, UK). Inhouse biochemical identification tests provided inconclusive results. Further identification using MALDI-TOF Mass Spectrometry (Bruker, Germany) and Kauffmann White agglutination identified the isolate as Salmonella Typhi which was typed to be lysotypeE1a. Susceptibility testing using VITEK-2 showed the isolate to be resistant to ampicillin, ampicillin/sulbactam, tobramycin and cotrimoxazole while being sensitive to piperacillin/tazobactam, cefotaxime, ceftriaxone, ceftazidime, cefpodoxime, imipenem, meropenem, ertapenem, ciprofloxacin (minimum inhibitory concentration $(\mathrm{MIC})=0.047 \mathrm{mg} / \mathrm{L}$ ), and moxifloxacin.

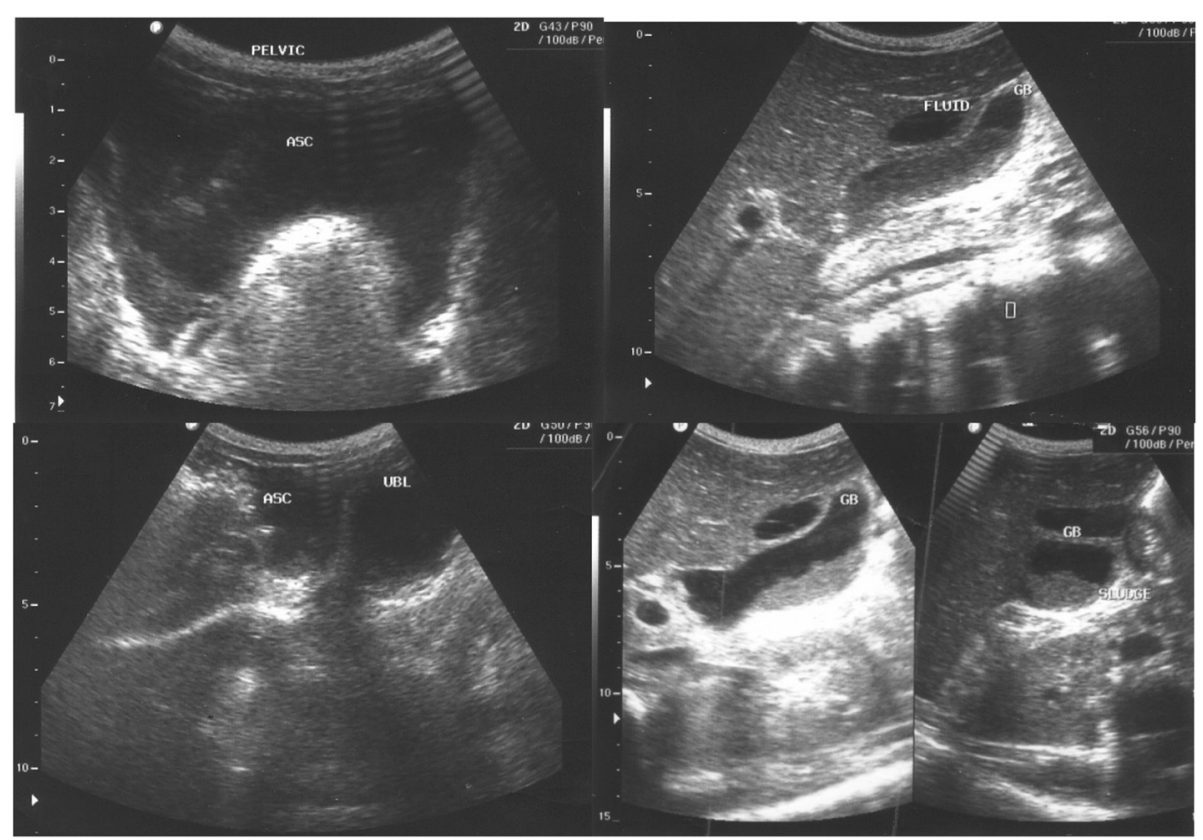

Figure 1 Ultra sound showing accumulation of fluid in the peritoneal cavity and dilated thickened wall gall bladder: features consistence with peritonitis and cholecystitis. 
The condition of the patient improved clinically on ceftriaxone, paracetamol and praziquantel $400 \mathrm{mg}$. Despite the patient being weak, he was discharged following relative's request on day 21 to be followed at pediatric outpatient clinics.

\section{Discussion}

Salmonella Typhi sepsis with meninges involvement is an uncommon complication in adults and older children $[1,12]$. Few cases have been reported in infants and have been associated with increased morbidity and mortality [4,6-10]. In the present case, a 9-year old boy was confirmed to have Salmonella Typhi meningitis based on CSF culture. Although this case presented at the beginning with symptoms suggestive of typhoid fever; the diagnosis of typhoid fever was not reached due nonspecific presentation of typhoid fever in endemic area; signifying the need for improved access to diagnostic microbiology in Africa [13]. Usually, typhoid fever presents with continual fever, accompanied by increasing fatigue, abdominal distension, constipation, abdominal pain, frontal headaches, malaise, anorexia, nausea and vomiting [14,15]. Later in the disease course the patient may present with jaundice due to elevated conjugated bilirubin as a result of endotoxin induced cholestasis [16]. All these features appeared in the present case at the district hospital. However, due to limited diagnostic tests and lack of high index of suspicion, lumber puncture and blood culture were not done. Though positive in our patient, the Widal test is of limited value in a hyperendemic country such as Tanzania since a large number of patients present with a positive Widal reaction due to previous infections.

Acute bacterial meningitis due to Salmonella Typhi is uncommon in adults and older children; however, when suspected, appropriate treatment should be initiated as early as possible to prevent associated morbidity and mortality. The diagnosis of typhoid fever at the district hospitals is a challenge in Tanzania due lack of diagnostic facilities. In addition the treatment is also a challenge because commonly available antibiotic for sepsis are ampicillin/amoxicillin and gentamicin of which most isolates causing sepsis are resistant. Moreover, gentamicin is usually not effective for facultative intracellular organisms such as salmonellae [17-19].

Although not proven, we cannot exclude a promoting effect on typhoid fever complicating meningitis by co-infection with Schistosoma haematobium in this child. A positive association between schistosomiasis and Salmonella Typhi has been described in patients as well as in experimental mouse models [20-23]; this could be explained by the argument that the parasite facilitates invasion via the urinary tract, so meningitis would follow bacteremia. Nevertheless; to date no case has been published associating urinary schistosomiasis and Salmonella Typhi meningitis; more research on this interaction is needed especially in endemic areas to confirm the argument.

Based on this case and other cases in infants and neonates, salmonella meningitis should be considered whenever gram-negative bacteria are seen in CSF in patients living in sub-Saharan Africa. Clinicians should have high index of suspicion especially if patients present with signs and symptoms of typhoid fever. The appropriate treatment for salmonella meningitis is a combination of ciprofloxacin (especially in adults) and ceftriaxone/cefotaxime [19]. Although chloramphenicol penetrates well through meninges, currently high resistance rates against this antibiotic to Salmonella Typhi argue against its use in sub-Saharan Africa [24]. In order to prevent relapse, high dose of a third-generation cephalosporin for at least 4 weeks is recommended $[19,25]$. In this case, history of low grade fever associated with abdominal distension which was followed by jaundice indicates that the disease started as typhoid fever with dissemination to the meninges. Lack of blood culture is the limitation to confirm this probable course.

\section{Conclusion}

We report this rare case to alert clinicians that Salmonella Typhi could contribute significantly as a possible etiology of bacterial meningitis in children in developing countries. The diagnosis requires proper investigation with high index of suspicion so that appropriate management can be instituted timely. Lastly, establishing bacteriological laboratories would largely contribute to rapidly differentiate between malaria and other bacteriological causes of diseases in developing countries.

\section{Consent}

Written informed consent was obtained from the patient's parents for publication of this Case Report and any accompanying images. A copy of the written consent is available for review by the Editor-in-Chief of this journal.

\section{Abbreviations}

ASC: Ascites; CSF: Cerebrospinal fluid; GB: Gall bladder; HIV: Human immunodeficiency virus; MIC: Minimum inhibitory concentration; TOF: Timeof-flight; UBL: Urinary bladder; UK: United Kingdom; WBC: White blood cells.

\section{Competing interests}

The authors declare that they have no competing interests.

\section{Authors' contributions}

FC and RK managed the patient and collected all clinical information. LG, SEM, MFM WS, OZ and UG performed laboratory procedures. FC, MMM, SEM and UG wrote the manuscript which was approved by all authors.

\section{Acknowledgment}

The authors would like to acknowledge all members of the department of Pediatric and Child Health Bugando Medical Centre, laboratory staff of 
Bugando Medical Centre and Institute of Medical Microbiology Goettingen. We thank the NRZ for Salmonella, Wernigerode, Germany for lysotyping.

\section{Author details}

${ }^{1}$ Department of Pediatric and child Health Weill Bugando School of Medicine, Catholic University of Health and Allied Sciences, Mwanza, Tanzania. ${ }^{2}$ Department of Microbiology and Immunology Weill Bugando School of Medicine, CUHAS-Bugando, P.O. BOX 1464, Mwanza, Tanzania. ${ }^{3}$ Institute of Medical Microbiology, University Medical Center Goettingen, Goettingen, Germany.

Received: 2 July 2014 Accepted: 23 February 2015

Published online: 03 March 2015

\section{References}

1. Feasey NA, Dougan G, Kingsley RA, Heyderman RS, Gordon MA. Invasive non-typhoidal salmonella disease: an emerging and neglected tropical disease in Africa. Lancet. 2012:379(9835):2489-99.

2. Graham SM. Salmonellosis in children in developing and developed countries and populations. Curr Opin Infect Dis. 2002;15(5):507-12.

3. Reddy EA, Shaw AV, Crump JA. Community-acquired bloodstream infections in Africa: a systematic review and meta-analysis. Lancet Infect Dis. 2010;10 (6):417-32.

4. Swann O, Everett DB, Furyk JS, Harrison EM, Msukwa MT, Heyderman RS, et al. Bacterial Meningitis in Malawian Infants2 Months of Age: Etiology and Susceptibility to World Health Organization First-Line Antibiotics. Pediatr Infect Dis J. 2014;33(6):560

5. Majowicz SE, Musto J, Scallan E, Angulo FJ, Kirk M, O'Brien SJ, et al. The global burden of nontyphoidal Salmonella gastroenteritis. Clin Infect Dis. 2010:50(6):882-9.

6. Karim M, Islam N. Salmonella meningitis: report of three cases in adults and literature review. Infection. 2002;30(2):104-8.

7. Nwadike V, Fowotade A, Tuta K, Olusanya O. A Rare Case of Salmonella typhi Meningitis in an Eleven Month Old Infant: A Case Report. Annals of Ibadan Postgraduate Medicine. 2012;10(1):38-9.

8. Cooke FJ, Ginwalla S, Hampton MD, Wain J, Ross-Russell R, Lever A, et al. Report of neonatal meningitis due to Salmonella enterica serotype Agona and review of breast milk-associated neonatal Salmonella infections. J Clin Microbiol. 2009:47(9):3045-9.

9. Adhikary R, Joshi S, Ramakrishnan M. Salmonella typhimurium meningitis in infancy. Indian journal of critical care medicine: peer-reviewed, official publication of Indian Society of Critical Care Medicine. 2013;17(6):392.

10. Appalaraju B, Parvathi S, Vijayalakshmi M. Fatal fulminant Salmonella infantis meningitis with septicemia. J Commun Dis. 2011;43(4):285-7.

11. OhAiseadha C, Dunne O, Desmond F, O'Connor M. Salmonella meningitis and septicaemia in an non-immunocompromised adult, associated with a cluster of Salmonella Enteritidis PT 14b, Ireland, November 2009. Euro Surveill. 2009;18(7):19489.

12. Parry CM. Epidemiological and clinical aspects of human typhoid fever Salmonella infections: clinical, immunological and molecular aspects. Cambridge: Cambridge University Press; 2006.

13. Petti CA, Polage CR, Quinn TC, Ronald AR, Sande MA. Laboratory medicine in Africa: a barrier to effective health care. Clin Infect Dis. 2006;42(3):377-82

14. Cunha BA. The death of Alexander the Great: malaria or typhoid fever? Infect Dis Clin North Am. 2004;18(1):53-63.

15. Malani PN. Mandell, Douglas, and Bennett's principles and practice of infectious diseases. JAMA. 2010:304(18):2067-71.

16. Ratnayake EC, Shivanthan C, Wijesiriwardena BC. Cholestatic hepatitis in a patient with typhoid fever-a case report. Ann Clin Microbiol Antimicrob. 2011;10:35.

17. Crump JA, Gove S, Parry CM. Management of adolescents and adults with febrile illness in resource limited areas. BMJ. 2011;343:d4847.

18. Mccracken J, Mize $S$, Threlkeld N. Intraventricular gentamicin therapy in gramnegative bacillary meningitis of infancy. Lancet. 1980;315(8172):787-91.

19. Price $\mathrm{EH}$, De Louvois J, Workman MR. Antibiotics for Salmonella meningitis in children. J Antimicrob Chemother. 2000;46(5):653-5.

20. Gendrel D, Richard-Lenoble D, Kombila M, Engohan E, Nardou M, Moussavou A, et al. Schistosoma intercalatum and relapses of Salmonella infection in children. Am J Trop Med Hyg. 1984;33(6):1166-9.

21. Gendrel D. Salmonella-Schistosoma interactions. Rev Prat. 1993;43(4):450-2.
22. Njunda A, Oyerinde J. Salmonella typhi infection in Schistosoma mansoni infected mice. West Afr J Med. 1995;15(1):24-30.

23. Rocha H, Kirk JW, Hearey CD. Prolonged Salmonella bacteremia in patients with Schistosoma mansoni infection. Arch Intern Med. 1971;128(2):254-7.

24. Groß U, Amuzu SK, De Ciman R, Kassimova I, Groß L, Rabsch W, et al. Bacteremia and antimicrobial drug resistance over time, Ghana. Emerg Infect Dis. 2011;17(10):1879.

25. Chiu C-H, Ou JT. Persistence of Salmonella species in cerebrospinal fluid of patients with meningitis following ceftriaxone therapy. Clin Infect Dis. 1999;28(5):1174-5.

\section{Submit your next manuscript to BioMed Central and take full advantage of:}

- Convenient online submission

- Thorough peer review

- No space constraints or color figure charges

- Immediate publication on acceptance

- Inclusion in PubMed, CAS, Scopus and Google Scholar

- Research which is freely available for redistribution

Submit your manuscript at www.biomedcentral.com/submit 